\title{
HYPERBOLIC DIMENSION OF METRIC SPACES
}

\author{
S. BUYALO AND V. SCHROEDER
}

To Viktor Abramovich Zalgaller

\begin{abstract}
A new quasi-isometry invariant of metric spaces, called the hyperbolic dimension (hypdim) is introduced; this is a version of Gromov's asymptotic dimension (asdim). The inequality hypdim $\leq$ asdim is always fulfilled; however, unlike the asymptotic dimension, hypdim $\mathbb{R}^{n}=0$ for every Euclidean space $\mathbb{R}^{n}$ (while $\operatorname{asdim} \mathbb{R}^{n}=n$ ). This invariant possesses the usual properties of dimension such as monotonicity and product theorems. The main result says that the hyperbolic dimension of any Gromov hyperbolic space $X$ (under mild restrictions) is at least the topological dimension of the boundary at infinity plus 1 , hypdim $X \geq \operatorname{dim} \partial_{\infty} X+1$. As an application, it is shown that there is no quasi-isometric embedding of the real hyperbolic space $\mathrm{H}^{n}$ into the metric product of $n-1$ metric trees stabilized by any Euclidean factor, $T_{1} \times \cdots \times T_{n-1} \times \mathbb{R}^{m}, m \geq 0$.
\end{abstract}

\section{$\S 1$. INTRODUCTION}

We introduce a new quasi-isometry invariant of metric spaces, called the hyperbolic dimension, hypdim, which is a version of Gromov's asymptotic dimension, asdim. We always have hypdim $\leq$ asdim; however, unlike the asymptotic dimension, hypdim $\mathbb{R}^{n}=0$ for every Euclidean space $\mathbb{R}^{n}$ (while asdim $\mathbb{R}^{n}=n$ ). This invariant possesses the usual properties of dimension such as monotonicity and product theorems. To formulate our main result, we recall that a metric space $X$ has bounded growth at some scale if for some constants $r, R$ with $R>r>0$, and some $N \in \mathbb{N}$ every ball of radius $R$ in $X$ can be covered by $N$ balls of radius $r$; see [BoS].

Theorem 1.1. Let $X$ be a geodesic Gromov hyperbolic space that has bounded growth at some scale and whose boundary at infinity $\partial_{\infty} X$ is infinite. Then

$$
\operatorname{hypdim} X \geq \operatorname{dim} \partial_{\infty} X+1 \text {. }
$$

As an application, we obtain the following.

Corollary 1.2. For every $n \geq 2$ there is no quasi-isometric embedding $\mathrm{H}^{n} \rightarrow T_{1} \times \cdots \times$ $T_{n-1} \times \mathbb{R}^{m}$ of the real $n$-dimensional hyperbolic space $\mathrm{H}^{n}$ into the product of $n-1$ trees stabilized by any Euclidean factor $\mathbb{R}^{m}, m \geq 0$.

Remark 1.3. For $n=2$ this result was proved in [BS2] by a different method.

Remark 1.4. In [BS2], for every $n \geq 2$ we constructed a quasi-isometric embedding of $\mathrm{H}^{n}$ into the $n$-fold product of homogeneous trees whose vertices have an infinite (countable) degree and whose edges have length 1. By Corollary 1.2, that embedding is optimal with

2000 Mathematics Subject Classification. Primary 54F45, 53C45.

Key words and phrases. Hyperbolic dimension, Gromov's asymptotic dimension.

The first author was supported by RFBR (grant no. 02-01-00090), by CRDF (grant no. RM1-2381ST-02), and by SNF (grant no. 20-668 33.01).

The second author was supported by the Swiss National Science Foundation. 
respect to the number of tree factors even if we allow stabilization by Euclidean factors. Furthermore, it follows that hypdim $\mathrm{H}^{n}=n$ for every $n \geq 2$.

Moreover, in [BDS] we constructed a quasi-isometric embedding $f: \Gamma \rightarrow T \times \cdots \times T$ of every Gromov hyperbolic group $\Gamma$ into the $n$-fold product of the binary tree $T$ with $n=\operatorname{dim} \partial_{\infty} \Gamma+1$. Thus, Theorem 1.1 implies (see Theorem 4.6 below) that the embedding $f$ is optimal with respect to the number of tree factors even if we allow stabilization by Euclidean factors.

Hyperbolic dimension versus subexponential corank. In our earlier paper [BS1] we introduced another quasi-isometry invariant of metric spaces, called the subexponential corank. This invariant gives an upper bound for the topological dimension of a Gromov hyperbolic space that can be quasi-isometrically embedded into a given metric space $X, \operatorname{rank}_{h}(X) \leq \operatorname{corank}(X)$. Thus, the corank is a useful tool for finding obstructions to such embeddings, and it works perfectly well in many cases; see [BS1] for the details. However, e.g., for quasi-isometric embeddings $\mathrm{H}^{n} \rightarrow T_{1} \times \cdots \times T_{k} \times \mathbb{R}^{m}$ it gives only $k \geq n-2$, while hypdim gives the optimal inequality $k \geq n-1$ by Corollary 1.2 . This drawback of the corank is closely related to the fact that $\operatorname{corank}(T)=1>0=\operatorname{dim} \partial_{\infty} T$ for every metric tree $T$, while $\operatorname{corank}(X)=\operatorname{dim} \partial_{\infty} X$ for every $\operatorname{CAT}(-1)$ Hadamard manifold $X$.

On the other hand, the hyperbolic dimension, which is perfect for a product of trees, is much harder to compute than the corank. For example, we do not even know the precise value of hypdim $\left(\mathrm{H}^{2} \times \mathrm{H}^{2}\right)$ (it must be 3 or 4 ). We also do not see any direct way to compare the corank and the hypdim. At the present stage of knowledge, these two invariants prove to be independent in a sense, each of them working perfectly well in its own range while failing in the other.

Structure of the paper. In $\S 2$ we introduce and discuss properties of the class of large scale doubling metric spaces. This class is a key ingredient of the hyperbolic dimension. The main result here is Proposition 2.8, which is an important step in the proof of Theorem 1.1.

In $\S 3$ we define the hyperbolic dimension and discuss its properties. $\S 4$ is devoted to the proof of Theorem 1.1 and Corollary 1.2.

Here we briefly recall some notions used in the body of the paper. We denote by $\left|x x^{\prime}\right|$ the distance in a metric space $X$ between $x, x^{\prime} \in X$. A map $f: X \rightarrow Y$ between metric spaces is quasi-isometric if for some $a \geq 1, b \geq 0$ we have

$$
\frac{1}{a}\left|x x^{\prime}\right|-b \leq\left|f(x) f\left(x^{\prime}\right)\right| \leq a\left|x x^{\prime}\right|+b,
$$

for every $x, x^{\prime} \in X$. In this case we also say that $f$ is $(a, b)$-quasi-isometric. A metric space is geodesic if every two points in it are connected by a geodesic. By a CAT(-1)-space we mean a complete, geodesic space whose triangles are thinner than the comparison triangles in the real hyperbolic plane $\mathrm{H}^{2}$.

\section{§2. LARGE SCALE DOUbling METRIC SPACES}

Here we introduce a class of metric spaces that will serve as a key ingredient in the introduction of the hyperbolic dimension.

2.1. Definition and properties. A subset $U$ of a metric space $X$ is large scale doubling if there is a constant $N \in \mathbb{N}$ such that for every sufficiently large $r>1$ and every ball $B_{2 r} \subset X$ of radius $2 r$, the intersection $B_{2 r} \cap U$ can be covered by at most $N$ balls of radius $r$. More precisely, we say that $U$ is $(N, R)$-large-scale doubling or $(N, R)$-ls-doubling if 
the above condition is fulfilled for all $r \geq R$. We also say that $U$ is $N$-ls-doubling if only $N$ is of importance.

An equivalent definition is that for all sufficiently large $r$ every intersection $B_{2 r} \cap U$ contains at most $N$ points that are $r$-separated.

Examples 2.1. 1. Any Euclidean space $\mathbb{R}^{n}$ is $(N, R)$-ls-doubling for some $N=N(n)$ and $R=0$.

2. Let $B$ be a bounded metric space. Then the metric product $X=B \times \mathbb{R}^{n}$ is $(N, R)$ ls-doubling for some $N=N(n)$ and $R \geq 2 \operatorname{diam} B$. We emphasize that in this example the parameter $N$ counting the number of separated points or of covering balls is actually independent of $B$, while the parameter $R$ describing the corresponding scales tends to infinity as $\operatorname{diam} B \rightarrow \infty$ if as $B$ we take, say, an $\mathbb{R}$-tree.

Lemma 2.2. Assume that $U \subset X$ is $(N, R)$-ls-doubling. Then for any $r \geq \rho \geq R$, every intersection $B_{r} \cap U$ is covered by at most $N^{n}$ balls $B_{\rho}$ with $n=\log _{2}(2 r / \rho)$. In particular, $B_{r} \cap U$ contains at most $N^{n}$ points that are $2 \rho$-separated.

Proof. Every intersection $B_{r} \cap U$ is covered by at most $N^{n}$ balls of radius $r / 2^{n}$. There exists $n \in \mathbb{N}$ with

$$
\frac{r}{2^{n}}<\rho \leq \frac{r}{2^{n-1}}
$$

Then $n \leq \log _{2} \frac{2 r}{\rho}$, which proves the first assertion. For the second, we note that any ball $B_{\rho}$ contains at most one point of any $2 \rho$-separated set.

The property of being large scale doubling is a quasi-isometry invariant.

Proposition 2.3. Let $f: X \rightarrow Y$ be a quasi-isometric map. If $U \subset X, V \subset Y$ are large scale doubling, then $f(U) \subset Y, f^{-1}(V) \subset X$ are large scale doubling quantitatively.

Proof. We may assume that $f$ is $(a, b)$-quasi-isometric for some $a \geq 1, b \geq 0$, and that $r \geq 2 b$. The inverse image of any ball $B_{2 r} \subset Y$ is contained in a ball $B_{R} \subset X$ of radius $R=a(4 r+b)$. The image of any ball $B_{\rho} \subset X$ of radius $\rho=\frac{1}{a}(r-b)$ is contained in a ball $B_{r} \subset Y$.

By Lemma 2.2, the intersection $B_{R} \cap U$ is covered by at most $N^{k}$ balls $B_{\rho}$ with

$$
k=\log _{2} \frac{2 R}{\rho}=\log _{2} \frac{2 a^{2}(4 r+b)}{r-b} \leq \log _{2} 18 a^{2} .
$$

Thus, the intersection $B_{2 r} \cap f(U)$ is covered by at most $N^{k}$ balls $B_{r}$, where the upper bound $N^{k}$ is independent of $r$.

Lemma 2.4. If $U \subset X, V \subset Y$ are large scale doubling, then $U \times V \subset X \times Y$ is large scale doubling quantitatively.

Proof. By Proposition 2.3, we can consider the $l_{\infty}$-product metric on $X \times Y$. Then $B_{r}((x, y))=B_{r}(x) \times B_{r}(y)$ for any $(x, y) \in X \times Y$ and $r>0$. Now the large scale doubling property of $U \times V$ is obvious.

2.2. Large scale doubling sets in CAT(-1)-spaces. Let $X$ be a CAT $(-1)$-space. We fix a base point $x_{0} \in X$ and define the angle metric $\angle_{\infty}$ on the boundary at infinity $\partial_{\infty} X$ as follows. Given $\xi, \xi^{\prime} \in \partial_{\infty} X$, we consider the unit speed geodesic rays $c_{\xi}, c_{\xi^{\prime}}$ from $x_{0}$ to $\xi, \xi^{\prime}$, respectively, and put

$$
\angle_{\infty}\left(\xi, \xi^{\prime}\right)=\lim _{s \rightarrow \infty} \angle\left(\bar{c}_{\xi}(s) \bar{o} \bar{c}_{\xi^{\prime}}(s)\right)
$$


where $\angle\left(\bar{c}_{\xi}(s) \bar{o} \bar{c}_{\xi^{\prime}}(s)\right)$ is the angle at $\bar{o}$ of the comparison triangle in $\mathrm{H}^{2}$ for the triangle $c_{\xi}(s) x_{0} c_{\xi^{\prime}}(s)$. By the parallelism angle formula, we have

$$
\tan \left(\frac{1}{4} \angle_{\infty}\left(\xi, \xi^{\prime}\right)\right)=e^{-\operatorname{dist}\left(\bar{o}, \bar{\xi} \bar{\xi}^{\prime}\right)}
$$

where $\bar{\xi}, \bar{\xi}^{\prime} \in \partial_{\infty} \mathrm{H}^{2}$ satisfy $\angle_{\bar{o}}\left(\bar{\xi}, \bar{\xi}^{\prime}\right)=\angle_{\infty}\left(\xi, \xi^{\prime}\right)$, and $\bar{\xi} \bar{\xi}^{\prime}$ is the geodesic in $\mathrm{H}^{2}$ with the endpoints $\bar{\xi}, \bar{\xi}^{\prime}$ at infinity. Thus, $\angle_{\infty}\left(\xi, \xi^{\prime}\right) \leq 4 e^{-\operatorname{dist}\left(\bar{o}, \bar{\xi} \bar{\xi}^{\prime}\right)}$.

The shadow of a set $A \subset X$ is a subset $\operatorname{sh}(A) \subset \partial_{\infty} X$ that consists of the ends $\xi$ of all rays $x_{0} \xi$ intersecting $A$ (so $\left.\operatorname{sh}\left(x_{0}\right)=\partial_{\infty} X\right)$. Given $\delta>0$, we define the angle $\delta$-measure ${ }_{\delta} A$ of $A$ by

$$
{ }_{\delta} A=\inf _{\mathcal{C}} \sum_{B \in \mathcal{C}} \operatorname{diam}(\operatorname{sh}(B))
$$

where the infimum is taken over all coverings $\mathcal{C}$ of $A$ by balls of radius at least $\delta$ in $X$.

Lemma 2.5. Given $N \in \mathbb{N}, R>1$, for every sufficiently large $\delta$ there is a positive constant $C$ depending only on $N$ and $\delta$ and such that if a subset $A \subset X$ is $(N, R)$-lsdoubling and $\operatorname{dist}\left(x_{0}, A\right) \geq c>\delta$, then

$$
\angle_{\delta} A \leq C \cdot e^{-c / 2}
$$

Proof. We take $\delta / 2 \geq R$. Then, by Lemma 2.2, every ball $B_{r} \subset X$ with $r>\delta / 2$ contains at most $N^{n}=d \cdot r^{k}$ points of $A$ that are $\delta$-separated, where $k=\log _{2} N$ and $d=(4 / \delta)^{k}$. Furthermore, we may assume that $e^{c / 2} \geq(c+1)^{k}$ for each $c>\delta$.

Let $A^{\prime} \subset A$ be a maximal $\delta$-separated subset. Then $A \subset \bigcup_{a \in A^{\prime}} B_{\delta}(a)$. For any ball $B_{\delta}(a), a \in A^{\prime}$, consider $\xi, \xi^{\prime} \in \operatorname{sh}\left(B_{\delta}(a)\right)$ with $\angle_{\infty}\left(\xi, \xi^{\prime}\right)$ arbitrarily close to $\operatorname{diam}\left(\operatorname{sh}\left(B_{\delta}(a)\right)\right)$. For simplicity, we assume that $\angle_{\infty}\left(\xi, \xi^{\prime}\right)=\operatorname{diam}\left(\operatorname{sh}\left(B_{\delta}(a)\right)\right)$, because the possible errors disappear in the final conclusion.

Then $\operatorname{diam}\left(\operatorname{sh}\left(B_{\delta}(a)\right)\right) \leq 4 e^{-\operatorname{dist}\left(\bar{o}, \bar{\xi} \bar{\xi}^{\prime}\right)}$, in the notation introduced above. We take $x \in x_{0} \xi \cap B_{\delta}(a), x^{\prime} \in x_{0} \xi^{\prime} \cap B_{\delta}(a)$ and consider the piecewise geodesic curve $\gamma$ in $X$ that consists of the geodesic rays $x \xi, x^{\prime} \xi^{\prime}$ and the segment $x x^{\prime}$. The curve $\gamma$ connects the points $\xi, \xi^{\prime}$ in $X$, and $\operatorname{dist}\left(x_{0}, \gamma\right) \geq \operatorname{dist}\left(x_{0}, B_{\delta}(a)\right)=\left|x_{0} a\right|-\delta$. Furthermore, $\operatorname{dist}\left(x_{0}, \gamma\right) \leq$ $\operatorname{dist}\left(\bar{o}, \bar{\xi} \bar{\xi}^{\prime}\right)$ by comparison with $\mathrm{H}^{2}$. Thus,

$$
\operatorname{diam}\left(\operatorname{sh}\left(B_{\delta}(a)\right)\right) \leq 4 e^{\delta-\left|x_{0} a\right|}
$$

and

$$
\angle_{\delta} A \leq \sum_{a \in A^{\prime}} \operatorname{diam}\left(\operatorname{sh}\left(B_{\delta}(a)\right)\right)
$$

Since $c>\delta$, for each $\tau \geq c+1$ the number of points in $A^{\prime}$ whose distances to $x_{0}$ lie in the interval $[\tau-1, \tau)$ does not exceed $d \cdot \tau^{k}$. Therefore, we have

$$
\begin{aligned}
{ }_{\delta} A & \leq 4 e^{\delta} \sum_{a \in A^{\prime}} e^{-\left|x_{0} a\right|} \leq 4 d e^{\delta}\left(\sum_{q=0}^{\infty}(c+q+1)^{k} e^{-q}\right) e^{-c} \\
& \leq 4 d e^{\delta}\left(\sum_{q=0}^{\infty}(q+1)^{k} e^{-q}\right)(c+1)^{k} e^{-c} \leq C \cdot e^{-c / 2}
\end{aligned}
$$

by the choice of $c$. 
2.3. A cut property of large scale doubling subsets. A subset $A$ of a metric space $X$ is said to be roughly connected if for some $\sigma>0$ and every $a, a^{\prime} \in A$ there is a sequence $a_{0}=a, \ldots, a_{k}=a^{\prime}$ in $A$ with $\left|a_{i} a_{i-1}\right| \leq \sigma, i=1, \ldots, k$. Such a sequence is called a rough path or a $\sigma$-path between $a$ and $a^{\prime}$, and we also say that $A$ is $\sigma$-connected. We say that a roughly connected subset $A$ of a geodesic space $X$ is cut-quasiconvex if there is $c>0$ such that for every $a, a^{\prime} \in A$ and every $x \in a a^{\prime}$, every rough path in $A$ between $a, a^{\prime}$ intersects the ball $B_{c}(x) \subset X$ of radius $c$ and centered at $x$. In this case we also say that $A$ is $c$-cut-convex, and $c$ is called the cut radius of $A$. Obviously, this property implies that every geodesic segment $a a^{\prime} \subset X$ with endpoints $a, a^{\prime} \in A$ lies in the $c$-neighborhood of $A$; i.e., the set $A$ is, in particular, quasiconvex. This justifies our terminology.

Proposition 2.6. Assume that a $\sigma$-connected subset $A$ of a $\mathrm{CAT}(-1)$-space $X$ is $(N, R)$ ls-doubling for some $N \in \mathbb{N}, R \in \mathbb{R}$. Then $A$ is cut-quasiconvex, and the cut radius $c$ depends only on $N, R$, and $\sigma, c=c(N, R, \sigma)$.

Proof. Fix a sufficiently large $\delta$ provided by Lemma 2.5. We may assume that $\delta \geq \sigma$. Next, we take $c^{\prime}>\delta$ such that $C \cdot e^{-c^{\prime} / 2}<\pi$, where $C$ is the constant in Lemma 2.5, and put $c=c^{\prime}+\delta$. Then $c=c(N, R, \sigma)$.

Assume that for some $a, a^{\prime} \in A$ there is a $\sigma$-path $\gamma$ in $A$ between $a, a^{\prime}$ that misses the ball $B_{c}\left(x_{0}\right) \subset X$ for some $x_{0} \in a a^{\prime}$. Then $A^{\prime}=\bigcup_{b \in \gamma} B_{\delta}(b)$ is a connected subset in $X$ containing $a$ and $a^{\prime}$, so that $\angle_{\delta} A^{\prime} \geq \pi$ for every $\delta \geq \sigma$.

On the other hand, $\operatorname{dist}\left(x_{0}, A^{\prime}\right) \geq c^{\prime}$, and we have $\angle_{\delta} A^{\prime} \leq C \cdot e^{-c^{\prime} / 2}<\pi$ by Lemma 2.5 . This is a contradiction showing that $A$ is cut-quasiconvex with the cut radius $c$.

Corollary 2.7. If a ball $B_{r}$ of radius $r$ in a $\mathrm{CAT}(-1)$-space $X$ is $(N, R)$-ls-doubling for some $N \in \mathbb{N}$ and $R \in \mathbb{R}$, then $r \leq c$ for some constant $c=c(N, R)$.

When discussing cut-quasiconvexity, we have used so far only the fact that any large scale doubling space has a polynomial growth rate (actually, a subexponential growth rate suffices). In what follows, the next proposition plays a key role, and we use in it the entire strength of the definition of ls-doubling spaces.

Proposition 2.8. Let $N \in \mathbb{N}$, and let $A$ be an $N$-ls-doubling subset in a CAT(-1)-space $X$. Then for every $\sigma>0$ the union $\partial_{\infty} A_{\sigma}$ of the boundaries at infinity of the $\sigma$-connected components of $A$ contains at most $N$ points.

Proof. Fix $x_{0} \in X$. The cut-quasiconvexity of any roughly connected component of $A$ implies that a tail of every geodesic ray $x_{0} \xi \subset X, \xi \in \partial_{\infty} A_{\sigma}$, lies in the $c$-neighborhood of $A$ for some $c>0$ depending only on the bounding parameters for $A$ and $\sigma$.

Given $\xi \in \partial_{\infty} X$, we denote by $\xi(t) \in x_{0} \xi$ the point with $\left|\xi(t) x_{0}\right|=t$. For every finite collection $\Lambda \subset \partial_{\infty} A_{\sigma}$, we have $\left|\xi(r) \xi^{\prime}(r)\right| \geq r+2 c$ for all sufficiently large $r$ and all different $\xi, \xi^{\prime} \in \Lambda$. Thus, in $B_{2 r}\left(x_{0}\right) \cap A$ we find at least $|\Lambda|$ points that are pairwise $r$-separated. This shows that $|\Lambda| \leq N$.

\section{$\S 3$. The HYPERBolic DIMENSION}

The hyperbolic dimension is a version of Gromov's asymptotic dimension (see [Gr, 1.E]) with the only difference that we take the ls-doubling sets for the role of "small" sets. As in [BD], we find it convenient to use the Lebesgue number of a covering in our definition instead of $d$-multiplicity as in [Gr, 1.E].

Recall that the Lebesgue number of a covering $\mathcal{U}$ of a metric space $X$ is the maximal radius $L(\mathcal{U})$ such that any (open) ball in $X$ of that radius is contained in some element of the covering,

$$
L(\mathcal{U})=\inf _{x \in X} \sup \{\operatorname{dist}(x, X \backslash U): U \in \mathcal{U}\}
$$


A covering $\mathcal{U}$ of a metric space $X$ is said to be uniformly large scale doubling or uniformly ls-doubling if there exists $N \in \mathbb{N}$ with the following properties:

- there exists $R \geq 0$ such that every element of the covering is $(N, R)$-ls-doubling;

- any finite union of elements of the covering is $N$-ls-doubling.

We also say that such a covering is uniformly $N$-ls-doubling or uniformly $(N, R)$-lsdoubling.

Remark 3.1. To get a better grip on the second property, which is rather strong, assume that $X$ is a CAT $(-1)$-space. Then by Proposition 2.8 , the boundary at infinity $\partial_{\infty} A_{\sigma} \subset$ $\partial_{\infty} X$ of the roughly connected components of any finite union $A=\bigcup_{i} U_{i}$ of elements $U_{i} \in \mathcal{U}$ has cardinality bounded above independently of the number of the elements.

Let $\mathcal{U}$ be a family of subsets in a space $X$. The multiplicity $m(\mathcal{U})$ of $\mathcal{U}$ is the maximal number of members of $\mathcal{U}$ with nonempty intersection. We say that a family $\mathcal{U}$ is disjoint if $m(\mathcal{U})=1$.

A covering $\mathcal{U}$ of $X$ is said to be colored if it is a union of $m \geq 1$ disjoint families, $\mathcal{U}=\bigcup_{a \in A} \mathcal{U}^{a},|A|=m$. In this case we also say that $\mathcal{U}$ is $m$-colored. Clearly, the multiplicity of an $m$-colored covering is at most $m$.

Definition 3.2. The hyperbolic dimension of a metric space $X$, hypdim $X$, is the minimal $n$ such that for every $d>0$ there is an open $(n+1)$-colored covering $\mathcal{U}$ of $X$ with $L(\mathcal{U}) \geq d$ which is uniformly large scale doubling.

3.1. Properties of the hyperbolic dimension. Here we list some important properties of the hyperbolic dimension. The following two properties are obvious from the definition:

- hypdim $X=0$ for every large scale doubling space $X$;

- hypdim $X \leq \operatorname{asdim} X$ for every metric space $X$.

(The asymptotic dimension of a metric space $X$, $\operatorname{asdim} X$, is defined precisely in the same way as the hyperbolic dimension, but instead of uniformly $N$-ls-doubling coverings we take coverings by sets with uniformly bounded diameter; see [Gr], [BD].) The above inequality can be strict. For example, asdim $\mathbb{R}^{n}=n$ for every $n \geq 0$, while hypdim $\mathbb{R}^{n}=0$ because $\mathbb{R}^{n}$ is a large scale doubling space.

- Monotonicity of the hyperbolic dimension

The following theorem implies, in particular, that the hyperbolic dimension is a quasiisometry invariant of a metric space.

Theorem 3.3. Let $f: X \rightarrow X^{\prime}$ be a quasi-isometric map between metric spaces $X$ and $X^{\prime}$. Then

$$
\operatorname{hypdim} X \leq \operatorname{hypdim} X^{\prime} .
$$

Proof. This follows straightforwardly, by using the definition of the hyperbolic dimension and Proposition 2.3.

Corollary 3.4. The hyperbolic dimension is a quasi-isometry invariant of metric spaces.

- Product theorem for the hyperbolic dimension

By using a standard argument from dimension theory, the following can be shown.

Theorem 3.5. For any metric spaces $X_{1}$ and $X_{2}$, we have

$$
\operatorname{hypdim}\left(X_{1} \times X_{2}\right) \leq \operatorname{hypdim} X_{1}+\operatorname{hypdim} X_{2} \text {. }
$$

A detailed discussion of the proof can be found in [BS3, Chapter 13]. 


\section{§4. Proof of Theorem 1.1}

4.1. $d$-multiplicity of a covering. Fix $d>0$. Recall that the $d$-multiplicity of a covering $\mathcal{U}$ of a metric space $X$ does not exceed $n$ if no ball of radius $d$ in $X$ meets more than $n$ elements of the covering (see [Gr, 1.E]). An auxiliary hyperbolic dimension $\operatorname{hypdim}^{\prime} X$ can be defined as the minimal $n$ such that for every $d>0$ there is $N \in \mathbb{N}$ and a uniformly $N$-ls-doubling covering $\mathcal{U}$ of $X$ with $d$-multiplicity at most $\leq n+1$.

Lemma 4.1. We have hypdim' $X \leq \operatorname{hypdim} X$ for every metric space $X$.

Proof. Let $\mathcal{U}=\left\{U_{j}\right\}_{j \in J}$ be a uniformly $N$-ls-doubling $(n+1)$-colored covering of $X$, $n=\operatorname{hypdim} X$, with Lebesgue number $L(\mathcal{U}) \geq 2 d$ for some $N \in \mathbb{N}$ and some $d>0$. As in [BD, Assertion 1], we define $V_{j}=U_{j} \backslash D_{d}\left(X \backslash U_{j}\right), j \in J$, where $D_{d}(A)$ is the metric $d$-neighborhood of $A \subset X$. Since $L(\mathcal{U}) \geq 2 d$, the collection $\mathcal{V}=\left\{V_{j}\right\}_{j \in J}$ is still a covering of $X$. Moreover, $\mathcal{V}$ is uniformly $N$-ls-doubling because $V_{j} \subset U_{j}$ for every $j \in J$. Furthermore, if a ball $B_{d}(x) \subset X$ meets some $V_{j}$, then $x \in U_{j}$. Thus, the $d$-multiplicity of $\mathcal{V}$ does not exceed $n+1$. Hence, the claim is proven.

4.2. Ends of uniformly $N$-ls-doubling coverings of $X$. We recall that a metric space $X$ has bounded geometry if there is $\rho_{X}>0$ and a function $M_{X}:(0, \infty) \rightarrow(0, \infty)$ such that every ball $B_{r} \subset X$ of radius $r>0$ contains at most $M_{X}(r)$ points that are $\rho_{X}$-separated.

Lemma 4.2. Let $X$ be a CAT(-1)-space with bounded geometry, and let $\mathcal{U}$ be a uniformly $N$-ls-doubling covering of $X$ with finite d-multiplicity, for some $N \in \mathbb{N}$ and a sufficiently large $d$. Furthermore, assume that the elements of $\mathcal{U}$ are $\sigma$-connected for some $\sigma \geq 10 d$. Then $\partial_{\infty} U \subset \partial_{\infty} X$ is finite for every $U \in \mathcal{U}$, and the number of different elements of $\mathcal{U}$ with infinite diameter is finite.

Proof. Since the covering $\mathcal{U}$ is uniformly $N$-ls-doubling, there is $R \in \mathbb{R}$ such that every element of the covering is $(N, R)$-ls-doubling. Then, by Proposition 2.6, every $U \in \mathcal{U}$ is cut-quasiconvex with cut radius $c>0$ depending only on $N, R$, and $\sigma$. By Proposition 2.8, we have $\left|\partial_{\infty} U\right| \leq N$ for every $U \in \mathcal{U}$.

We assume that $X$ has $\left(\rho_{X}, M_{X}\right)$-bounded geometry, and that the $d$-multiplicity of $\mathcal{U}$ does not exceed $n$ for $d \geq \rho=\rho_{X}$. Then we put $M_{0}=(n+1) \cdot N \cdot M_{X}(2 c)$ and assume that there are at least $M_{0}$ different elements of the covering $\mathcal{U}$ with infinite diameter. Since every finite union of elements of $\mathcal{U}$ is $N$-ls-doubling, there is a common point $\xi \in \partial_{\infty} X$ of $\partial_{\infty} U$ for at least $M_{0} / N$ different elements $U \in \mathcal{U}$.

We fix $x_{0} \in X$ and consider the geodesic ray $x_{0} \xi \subset X$. By the cut-quasiconvexity of $U$, a tail $x_{1} \xi \subset x_{0} \xi$ lies in the $c$-neighborhood of every $U$ selected above. Thus, for every $x \in x_{1} \xi$ the ball $B_{2 c}(x)$ intersects at least $(n+1) \cdot M_{X}(2 c)$ different elements of the covering. On the other hand, $B_{2 c}(x)$ can be covered by at most $M_{X}(2 c)$ balls of radius $\rho$. Hence, there is a ball $B_{\rho}\left(x^{\prime}\right) \subset X$ that intersects at least $n+1$ different elements of the covering. This is a contradiction, because $d \geq \rho$ and the $d$-multiplicity of the covering does not exceed $n$. Thus, there are at most $M_{0}-1$ different elements of $\mathcal{U}$ with infinite diameter.

\subsection{Radial contraction.}

Lemma 4.3. Let $\mathcal{W}$ be a locally finite collection of subsets of finite diameter in the ray $[0, \infty)$. Then there is a 1 -Lipschitz homeomorphism $f:[0, \infty) \rightarrow[0, \infty)$ such that every set $f(W), W \in \mathcal{W}$, has diameter at most 1 .

Proof. We put $r_{0}=[0, \infty)$ and let $V_{1}$ be the set of all $W \in \mathcal{W}_{0}=\mathcal{W}$ that intersect the initial segment of length 1 of $r_{0}$. Since the collection $\mathcal{W}$ is locally finite, $V_{1}$ is finite. 
Then $t_{1}=\sup \left\{t \in r_{0}: t \in W \in V_{1}\right\}$ is finite, and we define $\varphi_{1}: r_{0} \rightarrow r_{0}$ by $\varphi_{1}(t)=t$ for $t \in[0,1]$, and, for $t \geq 1$, by $\varphi_{1}(t)=t$ if $t_{1} \leq 2$ and $\varphi_{1}(t)=\frac{1}{t_{1}-1}(t-1)+1$ otherwise. Then $\varphi_{1}$ is a 1-Lipschitz homeomorphism, and $\operatorname{diam}\left(\varphi_{1}(W)\right) \leq 2$ for every $W \in V_{1}$.

We put $f_{1}=\varphi_{1}$ and note that $\mathcal{W}_{1}=f_{1}(\mathcal{W}) \cap r_{1}$ is a locally finite collection of subsets in the ray $r_{1}=[1, \infty)$. Now, we apply the same procedure to the ray $r_{1}$ and the collection $\mathcal{W}_{1}$, extending the resulting 1-Lipschitz homeomorphism $\varphi_{2}: r_{1} \rightarrow r_{1}$ to $r_{0}$ by the identity and putting $f_{2}=\varphi_{2} \circ f_{1}$.

Repeating, we obtain a stabilizing sequence of 1-Lipschitz homeomorphisms $f_{n}=$ $\varphi_{n} \circ f_{n-1}: r_{0} \rightarrow r_{0}, f_{n}=f_{n-1}$ on $[0, n]$, for which $\operatorname{diam}\left(f_{n}(W)\right) \leq 2$ for all $W \in \mathcal{W}$ intersecting $[0, n]$. Composing the limit homeomorphism $\lim _{n \rightarrow \infty} f_{n}$ with the homothety $\lambda(t)=t / 2$, we obtain the required homeomorphism $f$.

4.4. Cone over the boundary at infinity. The following estimate from below is the major step in the proof of Theorem 1.1.

Proposition 4.4. Let $X \subset \mathrm{H}^{n}, n \geq 2$, be the convex hull of a compact infinite set $Z \subset \partial_{\infty} \mathrm{H}^{n}$. Then hypdim $X \geq \operatorname{dim} Z+1$.

For the proof we need some preparations. We fix a base point $o \in X$ and define the cone over $Z, \operatorname{Co}(Z) \subset X$, as the union of all geodesic rays emanating from $o$ towards $Z$. Note that $\operatorname{Co}(Z)$ with the metric induced from $\mathrm{H}^{n}$ is, in general, neither $\operatorname{CAT}(-1)$ nor even a geodesic space, as for example in the case where $\operatorname{dim} Z=0$. On the other hand, $\mathrm{Co}(Z)$ is cobounded in $X$ (see [BoS, Proposition 10.1(2)]); that is, $\operatorname{dist}(x, \mathrm{Co}(Z)) \leq \sigma_{0}$ for some $\sigma_{0}>0$ and every $x \in X$. In what follows, we also use the angle metric in $\partial_{\infty} \mathrm{H}^{n}$ based at $o$.

Next, we consider the annulus $\operatorname{An}(Z) \subset \mathrm{Co}(Z)$ that consists of all $x \in \mathrm{Co}(Z)$ with $1 \leq|x o| \leq 2$. Clearly, $\operatorname{An}(Z)$ is homeomorphic to $Z \times I, I=[0,1]$. By a well-known result of dimension theory (see $[\mathrm{Al}]$ ), for the topological dimension we have

$$
\operatorname{dim} \operatorname{An}(Z)=\operatorname{dim} Z+1 \text {. }
$$

We also need the following simple fact from dimension theory.

Lemma 4.5. Let $Z$ be an infinite compact metric space. Then for every finite $A \subset Z$ and all sufficiently small $\varepsilon>0$ we have $\operatorname{dim}\left(Z \backslash D_{\varepsilon}(A)\right)=\operatorname{dim} Z$, where $D_{\varepsilon}(A)$ is the open $\varepsilon$-neighborhood of $A$.

Proof. Since $Z$ is infinite, we may assume that $n=\operatorname{dim} Z>0$. Let $Y \subset Z$ be the subset that consists of all points $z \in Z$ at which $Z$ has dimension $n$. It is well known (see [HW, Chapter IV.5]) that $\operatorname{dim} Y=n$; thus $Y$ cannot be finite, and $Y \not \subset D_{\varepsilon}(A)$ for all sufficiently small $\varepsilon>0$. The claim follows.

Proof of Proposition 4.4. The space $\mathrm{H}^{n}$ and, with it, its subspace $X$ certainly have a bounded geometry. Let $\rho_{\mathrm{H}^{n}}>0$ and $M_{\mathrm{H}^{n}}:(0, \infty) \rightarrow(0, \infty)$ be the corresponding bounding parameters. Assume that hypdim $X<\operatorname{dim} Z+1$. Then, moreover, $\operatorname{hypdim}^{\prime} X<\operatorname{dim} Z+1$ (see Subsection 4.1). Thus, for $d \geq \rho_{\mathrm{H}^{n}}$ there is $N \in \mathbb{N}$ and a uniformly $N$-ls-doubling covering $\mathcal{U}$ of $X$ with $d$-multiplicity not exceeding $\operatorname{dim} Z+1$. We fix $\sigma \geq 10 d$ and observe that taking $\sigma$-connected components of $\mathcal{U}$ changes neither the $N$-ls-doubling property nor $d$-multiplicity. Therefore, without loss of generality, we may assume that the elements of $\mathcal{U}$ are $\sigma$-connected. By Lemma 4.2, there are only finitely many elements $U \in \mathcal{U}$ with infinite diameter, and the boundary at infinity of each of them is finite.

We let $\mathcal{V}$ be the set of $\sigma$-connected components of the induced covering $\mathcal{U} \cap \operatorname{Co}(Z)$ of $\operatorname{Co}(Z)$. Then $\mathcal{V}$ is uniformly $N$-ls-doubling, and its $d$-multiplicity does not exceed $\operatorname{dim} Z+1$. The covering $\mathcal{V}$ of $\operatorname{Co}(Z)$ can be represented as the disjoint union $\mathcal{V}=\mathcal{V}_{0} \cup \mathcal{V}_{\infty}$ 
where $\mathcal{V}_{0}$ consists of all $V \in \mathcal{V}$ with finite diameter, and $\mathcal{V}_{\infty}$ consists of all $V \in \mathcal{V}$ with infinite diameter.

There are natural polar coordinates $x=(z, t), z \in Z, t \geq 0$, in $\operatorname{Co}(Z)$. Every 1Lipschitz homeomorphism $f:[0, \infty) \rightarrow[0, \infty)$ induces a 1-Lipschitz homeomorphism $F: \operatorname{Co}(Z) \rightarrow \operatorname{Co}(Z), F(z, t)=(z, f(t))$, which does not change the visual diameter $\operatorname{diam}(\operatorname{sh}(A))$ of any $A \subset \mathrm{Co}(Z)$.

Given $V \in \mathcal{V}_{0}$, let $W=W_{V} \subset[0, \infty)$ be the ray projection of $V$, i.e.,

$$
W=\{t \geq 0:(z, t) \in V \text { for some } z \in Z\} .
$$

Then diam $W<\infty$, and the collection $\mathcal{W}=\left\{W_{V}: V \in \mathcal{V}_{0}\right\}$ is locally finite in $[0, \infty)$, because $Z$ is compact and $\mathcal{V}_{0}$ is locally finite. By the radial contraction Lemma 4.3, there is a 1-Lipschitz homeomorphism $f:[0, \infty) \rightarrow[0, \infty)$ such that every set $f(W) \subset[0, \infty)$, $W \in \mathcal{W}$, has diameter at most 1 . For the induced homeomorphism $F: \operatorname{Co}(Z) \rightarrow \operatorname{Co}(Z)$ we denote by $\mathcal{V}^{1}$ the covering $F(\mathcal{V})=\{F(V): V \in \mathcal{V}\}$ of $\operatorname{Co}(Z)$. Then $\mathcal{V}^{1}=\mathcal{V}_{0}^{1} \cup \mathcal{V}_{\infty}^{1}$ for $\mathcal{V}_{0}^{1}=F\left(\mathcal{V}_{0}\right), \mathcal{V}_{\infty}^{1}=F\left(\mathcal{V}_{\infty}\right)$, and every $V \in \mathcal{V}_{0}^{1}$ lies in some annulus of width 1 centered at $o$.

Consider the sequence of contracting homeomorphisms $F_{k}: \mathrm{Co}(Z) \rightarrow \mathrm{Co}(Z)$ given by $F_{k}(z, t)=\left(z, \frac{1}{k} t\right),(z, t) \in \operatorname{Co}(Z), k \in \mathbb{N}$, and the corresponding sequence of coverings $\mathcal{V}^{k}=\mathcal{V}_{0}^{k} \cup \mathcal{V}_{\infty}^{k}$, where $\mathcal{V}^{k}=F_{k}\left(\mathcal{V}^{1}\right)$. Using Lemma 2.5 and the fact that $F_{k}$ does not change the visual diameter, we easily show that the diameter of the elements of $\operatorname{An}(Z) \cap \mathcal{V}_{0}^{k}$ vanishes as $k \rightarrow \infty$.

On the other hand, the angle measure of $U \backslash B_{c}(o), U \in \mathcal{U}$, is exponentially small in $c$ by Lemma 2.5. It follows that for every $\varepsilon>0$ the shadow of $U \backslash B_{c}(o)$ is contained in the $\varepsilon$ neighborhood of the finite set $\partial_{\infty} U$, provided $c$ is chosen sufficiently large, $\operatorname{sh}\left(U \backslash B_{c}(o)\right) \subset$ $D_{\varepsilon}\left(\partial_{\infty} U\right) \subset \partial_{\infty} \mathrm{H}^{n}$. Thus, for each $V \in \mathcal{V}_{\infty}$, the sequence $F_{k} \circ F(V) \cap \operatorname{An}(Z)$ converges as $k \rightarrow \infty$ to a finite union of segments $(z,[1,2]) \subset \operatorname{An}(Z), z \in Z_{V}$, where $Z_{V} \subset \partial_{\infty} U$ for $U \in \mathcal{U}, U \supset V$. Since there are only finitely many elements $U \in \mathcal{U}$ with infinite diameter, and every $V \in \mathcal{V}_{\infty}$ is contained in one of them, there is a finite $A \subset Z$ such that $Z_{V} \subset A$ for every $V \in \mathcal{V}_{\infty}$. Moreover, for every $\varepsilon>0$ there is $k_{\varepsilon} \in \mathbb{N}$ such that the compact set $\operatorname{An}_{\varepsilon}(Z)=\operatorname{An}\left(Z \backslash D_{\varepsilon}(A)\right)$ misses any $V \in \mathcal{V}_{\infty}^{k}$, and thus it is covered by elements of $\mathcal{V}_{0}^{k}, k \geq k_{\varepsilon}$. The multiplicity of the covering $\mathcal{V}_{0}^{k}$ of $\operatorname{An}_{\varepsilon}(Z)$ does not exceed $\operatorname{dim} Z+1$, and the diameter of its elements vanishes as $k \rightarrow \infty$. Therefore, $\operatorname{dim} \operatorname{An}_{\varepsilon}(Z) \leq \operatorname{dim} Z$. However, by $(*)$ and Lemma $4.5, \operatorname{dim} \operatorname{An}_{\varepsilon}(Z)=\operatorname{dim} Z+1$ for all sufficiently small $\varepsilon>0$, a contradiction.

\subsection{Proofs of Theorem 1.1 and Corollary 1.2 .}

Proof of Theorem 1.1. By [BoS, Theorem 1.1], the space $X$ is roughly similar to a convex subset of $\mathrm{H}^{n}$ for some integer $n$. Actually, there it was proved that $\partial_{\infty} X$ is homeomorphic to a compact set $Z \subset \partial_{\infty} \mathrm{H}^{n}$ such that $X$ is roughly similar to the convex hull of $Z$ in $\mathrm{H}^{n}$. Thus, we may assume that $X$ is the convex hull of some compact infinite set $Z \subset \partial_{\infty} \mathrm{H}^{n}$. Now, Proposition 4.4 completes the proof.

Proof of Corollary 1.2. In fact, we prove a more general result.

Theorem 4.6. For a Gromov hyperbolic space $X$ satisfying the conditions of Theorem 1.1, assume that there is a quasi-isometric embedding

$$
f: X \rightarrow T_{1} \times \cdots \times T_{k} \times Y
$$

into the $k$-fold product of metric trees $T_{1}, \ldots, T_{k}$ stabilized by a large scale doubling factor $Y$. Then $k \geq \operatorname{dim} \partial_{\infty} X+1$. 
Proof. First, we note that hypdim $Y=0$. Next, the asymptotic dimension of every metric tree $T$ is at most $1, \operatorname{asdim} T \leq 1$; see [DJ, Proposition 4]. Therefore, hypdim $T \leq 1$, and by the Product Theorem, the hyperbolic dimension of the target space is at most $k$. On the other hand, hypdim $X \geq \operatorname{dim} \partial_{\infty} X+1$ by Theorem 1.1 . Now, the required estimate follows from the monotonicity of hypdim; see Theorem 3.3.

\section{ACKNOWLEDGMENT}

We thank Kathrin Haltiner for useful discussions about large scale doubling sets. The first author is pleased to acknowledge the hospitality and support of the University of Zürich, where this research was carried out.

\section{REFERENCES}

[Al] P. Alexandroff, Dimensionstheorie. Ein Beitrag zur Geometrie der abgeschlossenen Mengen, Math. Ann. 106 (1932), 161-238.

[BD] G. Bell and A. Dranishnikov, On asymptotic dimension of groups acting on trees, Algebr. Geom. Topol. 1 (2001), 57-71 (electronic). MR1808331 (2001m:20062)

[BM] M. Bestvina and G. Mess, The boundary of negatively curved groups, J. Amer. Math. Soc. 4 (1991), no. 3, 469-481. MR1096169 (93j:20076)

[BoS] M. Bonk and O. Schramm, Embeddings of Gromov hyperbolic spaces, Geom. Funct. Anal. 10 (2000), no. 2, 266-306. MR1771428 (2001g:53077)

[BDS] S. Buyalo, A. Dranishnikov, and V. Schroeder, Embedding of hyperbolic groups into products of binary trees, Invent. Math. 169 (2007), no. 1, 153-192. MR2308852

[BS1] S. Buyalo and V. Schroeder, Hyperbolic rank and subexponential corank of metric spaces, Geom. Funct. Anal. 12 (2002), 293-306. MR1911661 (2003e:53045)

[BS2] - Embedding of hyperbolic spaces in the product of trees, Geom. Dedicata 113 (2005), 75-93. MR2171299 (2006f:53055)

[BS3] Elements of asymptotic geometry, EMS Monographs in Mathematics, 2007. European Mathematical Society (EMS), Zürich, 2007. MR2327160

[DJ] A. Dranishnikov and T. Januszkiewicz, Every Coxeter group acts amenably on a compact space, Proceedings of the 1999 Topology and Dynamics Conference (Salt Lake City, UT), Topology Proc. 24 (1999), Spring, 135-141. MR1802681 (2001k:20082)

[Gr] M. Gromov, Asymptotic invariants of infinite groups, Geometric Group Theory, Vol. 2 (Sussex, 1991) (G. A. Niblo, M. A. Roller, eds.), London Math. Soc. Lecture Note Ser., vol. 182, Cambridge Univ. Press, Cambridge, 1993, pp. 1-295. MR1253544 (95m:20041)

[HW] W. Hurewicz and H. Wallman, Dimension theory, Princeton Math. Ser., vol. 4, Princeton Univ. Press, Princeton, NJ, 1941. MR0006493 (3:312b)

[JS] T. Januszkiewicz and J. Swiatkowski, Hyperbolic Coxeter groups of large dimension, Comment. Math. Helv. 78 (2003), 555-583. MR1998394 (2004h:20058)

St. Petersburg Branch, Steklov Mathematical Institute, Russian Academy of Sciences, Fontanka 27, St. Petersburg 191023, Russia

E-mail address: sbuyalo@pdmi.ras.ru

Institut für Mathematik, Universität Zürich, Winterthurer Strasse 190, CH-8057, Zürich, SWITZERLAND

E-mail address: vschroed@math.unizh.ch

Received 10/OCT/2006

Translated by S. V. BUYALO 